Gefässchirurgie 2014 • 19:486-488

DOI 10.1007/s00772-014-1378-y

Online publiziert: 26. Juli 2014

(c) Springer-Verlag Berlin Heidelberg 2014

\author{
M. Werthern \\ Rechtsanwalt, München
}

\section{Kooperieren, honorieren, sanktionieren}

\author{
Honorar- und Konsiliarärzte in \\ der stationären Versorgung
}

\section{Ausgangslage, Problemstellung}

Bis 2007 waren die Sektorengrenzen zwischen ambulanter und stationärer Versorgung personell undurchlässig. Eine gleichzeitige Tätigkeit im Krankenhaus und in der ambulanten Versorgung war im Grundsatz genauso wenig möglich wie umgekehrt. Der Belegarzt bildete die Ausnahme. Den ersten Schritt zur Verzahnung beider Versorgungsbereiche tat der Gesetzgeber zum 01.01.2007 mit einer Änderung der Zulassungsverordnung für Vertragsärzte (Ärzte-ZV). Er hob den bis dahin geltenden Ausschluss einer Tätigkeit im Krankenhaus für Vertragsärzte auf.

Mit den Gesetzen „zur Weiterentwicklung der Organisationsstrukturen“ (GKV-OrgWG 2008) und zur „Verbesserung der Versorgungsstrukturen“ (GKVVStG 2011) und jüngst durch die Änderung des $\$ 2$ Abs.1 KHEntgG (2012) wurden die Möglichkeiten zur Kooperation zwischen ambulanter und stationärer Versorgung weiter ausgebaut. Gleichzeitig schuf der Gesetzgeber mit dem GKVVStG Sanktionen, um Missbräuche der neuen Kooperationsmöglichkeiten zu bekämpfen, die unter dem Schlagwort „Korruption im Gesundheitswesen "vor allem von den gesetzlichen Krankenkassen angeprangert werden.

》) Über 60\% der medizinischen Einrichtungen in Deutschland sind auf den Einsatz von Honorarärzten angewiesen
Über $60 \%$ der medizinischen Einrichtungen in Deutschland sind auf den Einsatz von Honorarärzten angewiesen, Tendenz steigend. Honorar- und Konsiliarärzte sind längst keine Lückenbüßer mehr, sondern für viele Krankenhäuser unverzichtbar, um den Versorgungsauftrag erfüllen zu können. Deshalb ist es von praktischer Relevanz, die rechtlichen Möglichkeiten und Grenzen des sektorenübergreifenden Einsatzes von Honorar- und Konsiliarärzten aufzuzeigen.

\section{Typologie Honorar-, Honorarbelegarzt, Konsiliararzt}

Nach der Begriffsbestimmung der Bundesärztekammer werden als Honorarärzte Fachärztinnen und Fachärzte bezeichnet, die in medizinischen Einrichtungen zeitlich befristet freiberuflich auf Honorarbasis tätig sind. Ärzte, die in Teilzeit oder bei Vermittlungsagenturen angestellt sind oder von diesen vermittelt werden (Arbeitnehmerüberlassung), sind nach Ansicht der Deutschen Krankenhausgesellschaft (DKG) nicht freiberuflich tätig und sind deshalb nicht als Honorarärzte anzusehen. Das hat Konsequenzen für das Liquidationsrecht des Arztes:

Nur der freiberufliche Honorararzt kann „Wahlarzt“ sein. Schaltet z. B. ein kommunaler Krankenhausträger eine krankenhauseigene „Personalservice $\mathrm{GmbH}^{\prime}$ ein, die Ärzte an das Krankenhaus vermittelt, so verliert der Arzt als angestellter Arzt der Personalvermittlungs- gesellschaft seine Freiberuflichkeit und damit die Möglichkeit, als Wahlarzt seine privatärztlichen Leistungen abzurechnen oder durch das Krankenhaus abrechnen zu lassen.

Der Honorar-Belegarzt ist 2009 eingeführt worden. Im Gegensatz zum klassischen Belegarzt steht dem Honorar-Belegarzt kein eigener direkter Vergütungsanspruch gegen den Patienten zu, sein Vertragspartner ist in der Regel das Krankenhaus. Er hat darauf zu achten, dass er der kassenärztlichen Vereinigung (KV) die Tätigkeit als Honorar-Belegarzt in der Belegabteilung anzeigt.

Der Belegarzt sollte mit dem Krankenhaus ein angemessenes Entgelt für die Überlassung von OP-Räumen und Personal vereinbaren. Ansonsten setzt er sich dem Verdacht nach $\$ 128$ Abs. 2 S. 3 SGB V der „unzulässigen Zusammenarbeit zwischen Leistungserbringern und Vertragsärzten“ aus. Die unentgeltliche oder verbilligte Überlassung von Geräten und Materialien sowie die unentgeltliche Überlassung von Räumlichkeiten oder Personal gelten als unzulässige Zuwendungen. Mit der Annahme solcher Zuwendungen verstößt der Arzt gegen vertragsärztliche Pflichten.

„Konsil“ bedeutet die Beratung zweier oder mehrerer Ärzte nach vorangegangener Untersuchung des Patienten zum Zweck der Diagnose und/oder zur Feststellung des Heilplans. In der Praxis umfasst der Begriff des Konsiliararztes seit langem wesentlich mehr: Der Konsiliararzt erbringt Krankenhausleistungen, die 
von den angestellten Krankenhausärzten fachlich nicht abgedeckt werden. So kann es haftungsrechtlich geboten sein, bei einer fachübergreifenden Operation das Operationsteam mit einem die vorhandene Fachkompetenz ergänzenden Konsiliararzt zu besetzen.

Dies zeigt das Beispiel der Patientin, die an einem fortgeschrittenen Endometriumkarzinom litt: Den Eingriff führten zwei Gynäkologen ohne Beteiligung eines Viszeralchirurgen durch. Nach starker Blutung wurde die Operation abgebrochen, das Karzinom nicht entfernt. Die um eine gutachterliche Stellungnahme gebetenen Fachgesellschaften DGAV und DGGG sahen darin einen groben Behandlungsfehler und ein Organisationsverschulden. Den Eingriff hätten die Gynäkologen nur zusammen mit einem Viszeralchirurgen als fachübergreifendes Operationsteam durchdurchführen dürfen.

\section{Vergütung stationärer Leistungen des Honorararztes im Krankenhaus}

Der Honorararzt kann nach $₫ 2$ Abs. 1 Krankenhausentgeltgesetz (KHEntgG) im und für das Krankenhaus voll- und teilstationäre Leistungen erbringen. Wie wird die honorarärztliche Tätigkeit angemessen vergütet? Soweit der Arzt gesetzlich versicherte Patienten im und für das Krankenhaus behandelt, erbringt er als „Erfüllungsgehilfe" des Krankenhauses eine allgemeine Krankenhausleistung gemäß $\$ 2$ Abs. 1 S. 1 KHEntgG, die mit dem Krankenhausentgelt abgegolten ist. Das Krankenhaus haftet für ihn. Es muss seine Tätigkeit versichern und angemessen vergüten. In der Vergütungsvereinbarung ist der Anschein einer erhöhten Vergütungen als verkappte Provision für die Zuweisung von Patienten an den Krankenhausträger zu vermeiden. Strafverfolgungsbehörden sehen in überdurchschnittlich hohen Vergütungen ein Indiz für eine „Unrechtsvereinbarung" zwischen dem Krankenhaus Geschäftsführer und dem Honorararzt. Maßstab ist weniger der EBM, da der Honorararzt nicht vertragsärztlich tätig wird, sondern die Vergütung der im Krankenhaus fest angestellten Ärzte.
Die Vergütung privatärztlicher Leistungen durch Honorarärzte kann ebenfalls in der Kooperationsvereinbarung zwischen Krankenhaus und Honorararzt als Beteiligungsvergütung analog der Vergütung im Chefarztvertrag beim Liquidationsvorbehalt durch das Krankenhaus geregelt werden. Beansprucht der Honorararzt hingegen ein eigenes Liquidationsrecht gegenüber Wahlleistungspatienten, betritt er unsicheren Boden:

Die Deutschen Krankenhausgesellschaft (DKG) geht in den Erläuterungen zum Honorararztmustervertrag davon aus, dass der Honorar-Wahlarzt bei einer Wahlleistungsvereinbarung in die externe Liquidationskette einbezogen ist, wenn er auf Veranlassung eines liquidationsberechtigten Arztes Leistungen erbringt.

Teile von Rechtsprechung und Literatur sehen das anders, eine höchstrichterliche Entscheidung zur privatärztlichen Abrechnungsbefugnis von Honorarärzten steht noch aus. Nach dem Vorsichtsgebot ist daher gegenwärtig dem Honorararzt dringend zu empfehlen, sich kein eigenes Liquidationsrecht einräumen zu lassen. Dafür spricht zusätzlich der haftungsrechtliche Aspekt, wonach bei einem eigenen Liquidationsrecht der Grundsatz gilt: „Wer liquidiert, haftet auch“. Sollte ausnahmsweise aus besonderen Gründen ein eigenes Liquidationsrecht gewünscht werden, muss der Honorararzt in der Wahlleistungsvereinbarung zwischen Krankenhaus und Patient als liquidationsberechtigter Arzt namentlich benannt werden. Nach einer jüngst ergangenen Entscheidung des LG Kiel berechtigt eine eigene Honorarvereinbarung mit dem Patienten nicht dazu, im oder für das Krankenhaus erbrachte Leistungen zu liquidieren, da sich das Liquidationsrecht (nur) aus der mit dem Krankenhaus getroffenen Wahlleistungsvereinbarung ableitet, soweit der Arzt darin namentlich genannt wird.

Das LG Würzburg sieht das genau anders herum: Es wies die Berufung eines Patienten zurück, der die Rückzahlung von Honorar verlangte, das er an den Honorararzt für dessen Operation gezahlt hatte. Begründung des Gerichts: Ein Rückzahlungsanspruch sei durch den zwischen den Parteien getroffenen Behandlungsvertrag ausgeschlossen, weil sich der Patient darin ausdrücklich zur Zahlung der liquidierten, nach GOÄ abgerechneten Vergütung verpflichtet habe. Als Konsequen $z$ aus beiden Entscheidungen sollte neben der Wahlleistungsvereinbarung des Patienten mit dem Krankenhaus eine weitere privatärztliche Vergütungsvereinbarung zwischen Honorararzt und Privatpatient geschlossen werden, wenn der Honorararzt mit Erfolg seine Vergütung direkt von dem Patienten verlangen will.

Soweit der Honorar-Wahlarzt nicht als ständiger ärztlicher Vertreter, sondern in sonstigen zulässigen Vertretungsfällen die Behandlung des Wahlleistungspatienten übernimmt, schließt $\$ 5$ Abs. 5 GOÄ die Berechnung von „Chefarztgebührensätzen" aus.

\section{Vergütung konsiliarärztlicher Leistungen}

Das Oberverwaltungsgericht (OVG) Lüneburg hat sich in einer aktuellen Entscheidung mit der Frage befasst, ob dem Erlösbudget eines kleineren Krankenhauses der Grundversorgung Stentimplantationen zugerechnet werden können, die nicht in dem Krankenhaus selbst, sondern in einem größeren etwa $20 \mathrm{~km}$ entfernten "Partnerkrankenhaus" von dort angestellten Ärzten durchgeführt wurden. Die Patienten wurden dafür an ein- und demselben Tag von dem Krankenhaus der Grundversorgung in das Partnerkrankenhaus und wieder zurücktransportiert.

Die Antwort auf die Frage hängt davon ab, ob es sich bei der Stentimplantation im Partnerkrankenhaus um eine Hauptleistung handelt oder lediglich um eine in den Therapieplan des abgebenden Krankenhauses integrierte ergänzende oder unterstützende Nebenleistung.

\section{》) Bei Gefäßerkrankungen ist die Abgrenzung zwischen "Verlegung" und „Verbringung" schwierig}

Das OVG hat die innerhalb eines Tages ohne stationäre Aufnahme des $\mathrm{Pa}$ tienten durchgeführte Stentimplantation als eine Nebenleistung angesehen, die dem Erlösbudget des abgebenden Krankenhauses zugerechnet werden kann: ein Grenzfall, der bei geringer Verschiebung 
in der Gewichtung der Abgrenzungskriterien auch zu einem anderen Ergebnis führen kann. Gerade bei Gefäßerkrankungen ist die Abgrenzung zwischen „Verlegung“ und „Verbringung“ schwierig. Aus Sicht des aufnehmenden Krankenhauses wird man darauf achten müssen, dass die Verantwortung für die weitere Behandlung übernommen und der Patient in die stationären Abläufe des Krankenhauses vollständig integriert wird.

\section{Fazit für die Praxis}

- Rechtlich und abrechnungstechnisch ist eine Teilanstellung des Arztes im Krankenhaus der Honorararzttätigkeit vorzuziehen.

- Die mit einer Teilanstellung verbundenen Nachteile wie Lohnnebenkosten, Einbindung in den Organisationsablauf des Krankenhauses, Verpflichtung zu festen Arbeitszeiten und Be- reitschaftsdienste sprechen hingegen für eine Honorararzttätigkeit.

- Honorarärzte müssen nicht ausschließlich freiberuflich tätig sein. Berufsrechtlich ist eine Honorararzttätigkeit im Krankenhaus neben der vertragsärztlichen Praxis zulässig, soweit damit keine unzulässige Zuweisung gegen Entgelt verbunden wird.

- Der Vertragsarzt mit vollem Versorgungsauftrag hat die zeitliche Obergrenze von 13 Wochenstunden bei seiner Tätigkeit im Krankenhaus zu beachten. Das wird von den Kassenärztlichen Vereinigungen (KVen) überprüft.

- Wenn der Honorararzt die Leitung und Organisation einer Abteilung in einem Krankenhaus neben seiner Praxis übernehmen will, sollte er erwägen, den hälftigen Versorgungsauftrag in seiner Praxis auf einen angestellten Arzt zu übertragen.
- Zweifel über die Sozialversicherungspflicht wegen „Scheinselbstständigkeit" beseitigt eine Anfrage bei der Clearingstelle der deutschen Rentenversicherung.

- Um Zweifel über die berufsrechtliche und vertragsärztliche Unbedenklichkeit der Honorararztvereinbarung vor Beginn der Tätigkeit auszuräumen, ist die Vorlage des Vertrages bei der Landesärztekammer und bei Vertragsärzten zusätzlich bei der KV zu empfehlen.

\section{Korrespondenzadresse}

\section{Werthern}

Rechtsanwalt

Keplerstraße 1, 81679 München

info@rawerthern.com

Interessenkonflikt. Der korrespondierende Autor gibt an, dass kein Interessenkonflikt besteht.

\section{Hier steht eine Anzeige.}

